This item was submitted to Loughborough's Research Repository by the author.

Items in Figshare are protected by copyright, with all rights reserved, unless otherwise indicated.

\title{
Electron kinetic effects in atmospheric dielectric-barrier glow discharges
}

PLEASE CITE THE PUBLISHED VERSION

PUBLISHER

(C) American Institute of Physics

VERSION

VoR (Version of Record)

LICENCE

CC BY-NC-ND 4.0

REPOSITORY RECORD

Zhu, X.M., and Michael G. Kong. 2019. "Electron Kinetic Effects in Atmospheric Dielectric-barrier Glow Discharges". figshare. https://hdl.handle.net/2134/5173. 
This item was submitted to Loughborough's Institutional Repository (https://dspace.lboro.ac.uk/) by the author and is made available under the following Creative Commons Licence conditions.

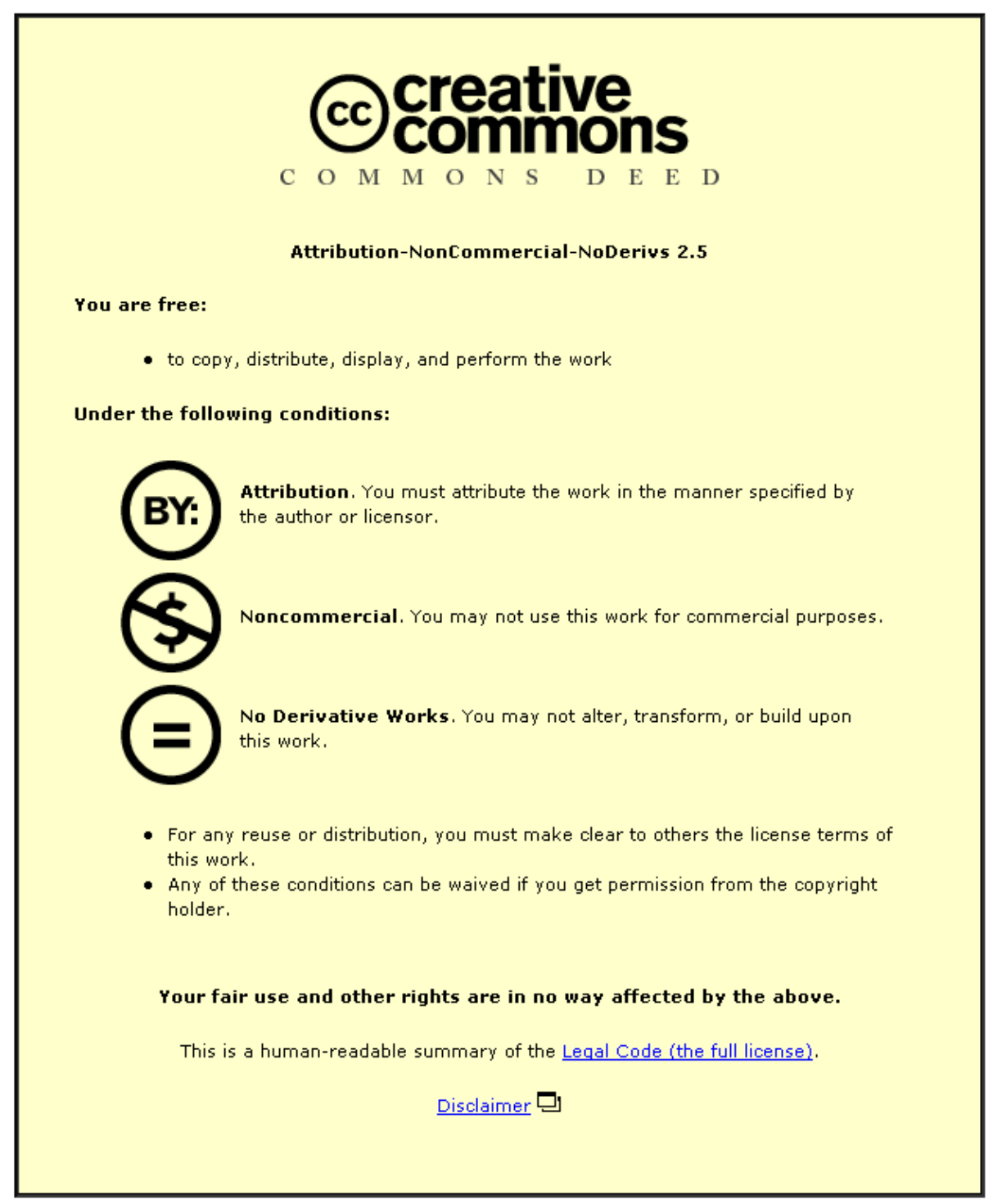

For the full text of this licence, please go to: http://creativecommons.org/licenses/by-nc-nd/2.5/ 


\title{
Electron kinetic effects in atmospheric dielectric-barrier glow discharges
}

\author{
X. M. Zhu and M. G. Konga) \\ Department of Electronic and Electrical Engineering Loughborough University, Ashby Road, Leicestershire \\ LE11 3TU, United Kingdom
}

(Received 9 June 2004; accepted 26 January 2005; published online 6 April 2005)

\begin{abstract}
Large-volume atmospheric dielectric-barrier discharges (DBD) are particularly useful for processing applications when they operate in their homogeneous mode. A vast majority of their theoretical studies is currently based on the hydrodynamic treatment in which electrons are assumed to be in equilibrium with the local electric field. Recognizing that this assumption is incorrect in the sheath region, we report the development of an electron-hybrid model to treat electrons kinetically and all other particles hydrodynamically. Through numerical examples, it is shown that the mainstream hydrodynamic model underestimates gas ionization and discharge current. Using the hybrid model, it is demonstrated that variation in the amplitude of the applied voltage does not significantly alter sheath characteristics in terms of the electric field and the electron mean energy. Also gas ionization in atmospheric DBD is found to be significant only over a short timescale of $1 \mu \mathrm{s}$. Compared with dc atmospheric pressure glow discharges, atmospheric DBD are shown to have a smaller electron mean energy and a larger sheath thickness. (C) 2005 American Institute of Physics.
\end{abstract}

[DOI: 10.1063/1.1872192]

\section{INTRODUCTION}

Atmospheric pressure glow discharges (APGD) have been a subject of active research because of their immense potentials for numerous processing applications including surface modification, pollution control, and sterilization. ${ }^{1-3}$ Their generation has been achieved over a broad spectrum from dc to microwave $\mathrm{e}^{2,4-6}$ and so far the most studied APGD are atmospheric dielectric-barrier discharges (DBD) generated at $1-100 \mathrm{kHz}$. Atmospheric DBD are nonthermal capacitive plasmas employing dielectrically coated electrodes. In their diffuse mode, they have a homogeneous visual appearance free of nanosecond filaments and their gas temperature can be kept below $100{ }^{\circ} \mathrm{C} .{ }^{7}$ Over the past five years, considerable advance has been made in both their scientific understanding and their technological development benefiting greatly from a series of theoretical studies of plasma dynamics. ${ }^{8-10}$ It is widely recognized that theoretical studies of APGD are increasingly indispensable to their future development.

Most theoretical investigations of atmospheric DBD are based on the hydrodynamic treatment in which electrons are assumed to be in equilibrium with the local electric field. When the discharge current density is modest, hydrodynamic simulation of atmospheric DBD yields good predictions of global parameters such as discharge current and voltage. ${ }^{8}$ On the other hand, it is known that APGD has a narrow sheath often less than $100 \mu \mathrm{m}$ and the electric field at the cathode surface usually exceeds $10 \mathrm{kV} / \mathrm{cm}^{8,9}$ For electrons emitting from the cathode surface, their initial kinetic energy is in the region of $0.5-1 \mathrm{eV}$ (Ref. 11) and so they are not in equilibrium with the local electric field for a substantial part of the sheath region. ${ }^{11,12}$ This shortcoming of the hydrodynamic model was recently highlighted for dc APGD (Ref. 12) and

${ }^{a)}$ Corresponding author; electronic mail: m.g.kong@lboro.ac.uk can cause gross errors in its description of electron kinetics in any APGD. Given that sheath characteristics and electron kinetics are key factors in determining both plasma chemistry and plasma stability, it is important to accurately describe electron kinetics particularly in the sheath region.

A complete description of kinetic effects usually requires Monte Carlo simulation. For APGD, the elevated gas pressure requires a vast number of computational super-particles and the CPU time needed is prohibitively high on today's computer workstation. As a first step toward a full account of kinetic effects in APGD, this article considers electron kinetics only and assumes that ions are in equilibrium with the local electric field. Therefore our approach is a hybrid plasma model for atmospheric DBD in which electrons are treated kinetically and all other plasma species (e.g., ions and metastables) are described hydrodynamically. This is in principle identical to electron-hybrid models used in lowpressure glow discharges. ${ }^{13}$ Its development is built on our previous work ${ }^{12}$ and is similar to that used recently for radiofrequency APGD. ${ }^{11}$ It is worth mentioning that the hybrid approach has achieved quantitative agreement with most available experimental data for DC APGD (Ref. 12) and radio-frequency APGD. ${ }^{11}$ So it is appropriate for simulation of atmospheric glow discharges. In Sec. II, an introduction is presented to a hybrid model and also to the hydrodynamic model. Section III presents the application of the hybrid model to atmospheric DBD and compares its results with that of the hydrodynamic model. Comparison between the two plasma models is made in terms of current-voltage characteristics, densities of charged and neutral species, and electric field. Additional insight is provided by the profile of the electron mean energy computed with the hybrid model. These are important to understand plasma stability and to optimize APGD applications. Using the hybrid model, we study in Sec. IV sheath characteristics including sheath dy- 
TABLE I. Electron-hybrid model: reactions considered for a He atmospheric DBD and their reaction rates.

\begin{tabular}{|c|c|c|}
\hline No & Reaction & Reaction rate \\
\hline \multicolumn{3}{|l|}{ Ionization } \\
\hline $\mathrm{R} 1$ & $e+\mathrm{He} \rightarrow \mathrm{He}^{+}+2 e$ & $2.584 \times 10^{-12} T_{\mathrm{e}}^{0.68} \exp \left(-2.854 \times 10^{5} / T_{\mathrm{e}}\right)$ \\
\hline $\mathrm{R} 2$ & $e+\mathrm{He}^{*} \rightarrow \mathrm{He}^{+}+2 e$ & $4.661 \times 10^{-10} T_{\mathrm{e}}^{0.60} \exp \left(-5.546 \times 10^{4} / T_{\mathrm{e}}\right)$ \\
\hline $\mathrm{R} 3$ & $e+\mathrm{He}_{2}^{*} \rightarrow \mathrm{He}_{2}^{+}+2 e$ & $1.268 \times 10^{-12} T_{\mathrm{e}}^{0.71} \exp \left(-3.945 \times 10^{4} / T_{\mathrm{e}}\right)$ \\
\hline \multicolumn{3}{|l|}{ Excitation } \\
\hline $\mathrm{R} 4$ & $\mathrm{He}+e \rightarrow \mathrm{He}^{*}+e$ & $2.308 \times 10^{-10} T_{\mathrm{e}}^{0.31} \exp \left(-2.297 \times 10^{5} / T_{\mathrm{e}}\right)$ \\
\hline R5 & $\mathrm{He}^{*}+2 \mathrm{He} \rightarrow \mathrm{He}_{2}^{*}+\mathrm{He}$ & $1.3 \times 10^{-33} \mathrm{~cm}^{6} / \mathrm{s}$ \\
\hline \multicolumn{3}{|c|}{ Deexcitation } \\
\hline R6 & $\mathrm{He}^{*}+e \rightarrow \mathrm{He}+e$ & $1.099 \times 10^{-11} T_{\mathrm{e}}^{0.31}$ \\
\hline \multicolumn{3}{|c|}{ Stepwise ionization } \\
\hline $\mathrm{R} 7$ & $\mathrm{He}^{*}+\mathrm{He}^{*} \rightarrow \mathrm{He}+\mathrm{He}^{+}+e$ & $2.7 \times 10^{-10} \mathrm{~cm}^{3} / \mathrm{s}$ \\
\hline \multicolumn{3}{|c|}{ Charge transfer } \\
\hline $\mathrm{R} 8$ & $\mathrm{He}^{+}+2 \mathrm{He} \rightarrow \mathrm{He}_{2}^{+}+\mathrm{He}$ & $1.0 \times 10^{-31} \mathrm{~cm}^{6} / \mathrm{s}$ \\
\hline \multicolumn{3}{|c|}{ Recombination } \\
\hline R9 & $\mathrm{He}_{2}^{+}+e \rightarrow \mathrm{He}^{*}+\mathrm{He}$ & $5.386 \times 10^{-7} T_{\mathrm{e}}^{0.5}$ \\
\hline
\end{tabular}

namics and comparison with dc APGD and radio-frequency APGD. Finally in Sec. V, our findings are summarized and discussed.

\section{ELECTRON HYBRID APGD MODEL}

We consider atmospheric DBD induced and sustained between two parallel-plate electrodes, each coated with a dielectric layer and connected externally to a kilovolt sinusoidal voltage source at audio frequencies. The background gas is atmospheric helium at $293 \mathrm{~K}$. Our model considers six species, namely, electrons $e$, helium ions $\mathrm{He}^{+}$, excited helium atoms $\mathrm{He}^{*}$, molecular helium ions $\mathrm{He}_{2}^{+}$, excited helium molecules $\mathrm{He}_{2}^{*}$, and background helium atoms He. Among these plasma species, there are nine chemical reactions including direct ionization, excitation, deexcitation, charge transfer from atomic helium ions to dimer helium ions, the step-wise ionization through $\mathrm{He}^{*}$. These are shown in Table I and all reaction rates are identical to those used in Ref. 11.

Our nonequilibrium plasma model is a self-consistent and continuum model. Its governing equations consist of the mass conservation equations to determine densities of each plasma species, the current continuity equation for calculation of the electric field, and the electron energy conservation equation for the electron mean energy. The electron energy equation is used to eliminate the need to relate rates of ionization and other chemical reaction to the local electric field thus removing the hydrodynamic assumption. Our model is one dimensional with the governing equations solved in the direction perpendicular to the electrode plane. Specifically the governing equations are

$$
\begin{aligned}
& \frac{\partial n_{e}}{\partial t}=-\frac{\partial \Gamma_{e}}{\partial x}+K_{i, j}(\varepsilon) n_{i} n_{j}, \\
& \frac{\partial n_{+, *}}{\partial t}=-\frac{\partial \Gamma_{+, *}}{\partial x}+K_{i, j}(\varepsilon) n_{i} n_{j}, \\
& J(t)=\varepsilon_{0} \frac{\partial E}{\partial t}-\left(-e \Gamma_{e}+e \sum_{p} \Gamma_{+, p}\right),
\end{aligned}
$$

$$
\begin{aligned}
\frac{\partial\left(n_{e} \varepsilon\right)}{\partial t}= & -\frac{\partial \Gamma_{\varepsilon}}{\partial x}+e \Gamma_{e} E-K_{L, i j}(\varepsilon) n_{i} n_{j} \\
& -3 \frac{m_{e}}{m_{\text {neut }}} N K_{\mathrm{mt}} n_{e} k\left(T_{e}-T_{\text {neut }}\right),
\end{aligned}
$$

where $n$ and $\Gamma$ are the density and flux of species, $\varepsilon$ the electron mean energy, and $J$ the current density. $K_{i j}$ and $K_{L, i j}$ are, respectively, the reaction rate and the energy gain rate due to a reaction between species $i$ and $j . K_{\mathrm{ml}}$ is the momentum transfer frequency corresponding to the elastic collision between electrons and background gas atoms. $D$ is the diffusion coefficient, $\mu$ the mobility, and $E$ the electric field. $m$ is the mass of a plasma species and $T$ is the temperature of a plasma species. Subscripts $e,+,+,{ }^{*}$, and neut denote, respectively, electrons, ions, metastables, and neutral particles. $p$ represents different ions considered in the model. Fluxes of all plasma species are given below

$$
\begin{aligned}
& \Gamma_{e}=-D_{e}(\varepsilon) \frac{\partial n_{e}}{\partial x}-\mu_{e} n_{e} E, \\
& \Gamma_{+}=-D_{+} \frac{\partial n_{+}}{\partial x}+\mu_{+} n_{+} E, \\
& \Gamma_{*}=-D_{*} \frac{\partial n_{*}}{\partial x}, \\
& \Gamma_{\varepsilon}=\frac{5}{3} \Gamma_{e} \varepsilon-n_{e} D_{e}(\varepsilon) \frac{\partial \varepsilon}{\partial x} .
\end{aligned}
$$

Transport properties are identical to that used in Ref. 11. Importantly the ionization coefficient, $K_{i j}$ and $K_{L, i j}$ (rate coefficients) are calculated now as a function of the electron mean energy rather than the local electric field. Also electron diffusion coefficient is set at $D_{e}(\varepsilon)=1737\left(T_{e} / 17406\right)$.

The boundary conditions for electrons at the surface of both electrodes are 
TABLE II. Hydrodynamic model: Reactions considered for a He atmospheric DBD and their reaction rates.

\begin{tabular}{|c|c|c|c|}
\hline No & Reaction & Reaction rate & Reference \\
\hline \multicolumn{4}{|c|}{ Direct ionization } \\
\hline $\mathrm{H} 1$ & $\mathrm{He}+\mathrm{e} \rightarrow \mathrm{He}^{+}+e+\mathrm{e}$ & $\alpha$ & 8 \\
\hline \multicolumn{4}{|l|}{ Excitation } \\
\hline $\mathrm{H} 2$ & $\mathrm{He}+\mathrm{e} \rightarrow \mathrm{He}^{*}+e$ & $\beta$ & 8 \\
\hline $\mathrm{H} 3$ & $\mathrm{He}^{*}+2 \mathrm{He} \rightarrow \mathrm{He}_{2}^{*}+\mathrm{He}$ & $1.9 \times 10^{-34} \mathrm{~cm}^{5} / \mathrm{s}$ & 14 \\
\hline \multicolumn{4}{|c|}{ Deexcitation } \\
\hline $\mathrm{H} 4$ & $\mathrm{He}^{*}+e \rightarrow \mathrm{He}+e$ & $4.2 \times 10^{-9} \mathrm{~cm}^{3} / \mathrm{s}$ & 15 \\
\hline \multicolumn{4}{|c|}{ Stepwise ionization } \\
\hline H5 & $\mathrm{He}^{*}+\mathrm{He}^{*} \rightarrow \mathrm{He}+\mathrm{He}^{+}+e$ & $2.9 \times 10^{-9} \mathrm{~cm}^{3} / \mathrm{s}$ & 14 \\
\hline H6 & $\mathrm{He}^{*}+\mathrm{He}_{2}^{*} \rightarrow \mathrm{He}_{2}^{+}+\mathrm{He}+e$ & $2.5 \times 10^{-9} \mathrm{~cm}^{3} / \mathrm{s}$ & 16 \\
\hline $\mathrm{H} 7$ & $\mathrm{He}_{2}^{*}+\mathrm{He}_{2}^{*} \rightarrow \mathrm{He}_{2}^{+}+2 \mathrm{He}+e$ & $1.3 \times 10^{-10} \mathrm{~cm}^{3} / \mathrm{s}$ & 16 \\
\hline \multicolumn{4}{|c|}{ Charge transfer } \\
\hline H8 & $\mathrm{He}^{+}+2 \mathrm{He} \rightarrow \mathrm{He}_{2}^{+}+\mathrm{He}$ & $6.3 \times 10^{-32} \mathrm{~cm}^{6} / \mathrm{s}$ & 15 \\
\hline \multicolumn{4}{|c|}{ Recombination } \\
\hline H9 & $\mathrm{He}^{+}+e \rightarrow \mathrm{He}$ & $2.0 \times 10^{-12} \mathrm{~cm}^{3} / \mathrm{s}$ & 17 \\
\hline H10 & $\mathrm{He}_{2}+e \rightarrow \mathrm{He}_{2}^{*}$ & $5.0 \times 10^{-10} \mathrm{~cm}^{3} / \mathrm{s}$ & 18 \\
\hline H11 & $\mathrm{He}^{+}+e+\mathrm{He} \rightarrow \mathrm{He}+\mathrm{He}^{+}$ & $1.0 \times 10^{-27} \mathrm{~cm}^{6} / \mathrm{s}$ & 17 \\
\hline H12 & $\mathrm{He}_{2}^{+}+2 e \rightarrow \mathrm{He}^{*}+e$ & $7.1 \times 10^{-20} \mathrm{~cm}^{6} / \mathrm{s}$ & 19 \\
\hline H13 & $\mathrm{He}_{2}^{+}+e \rightarrow \mathrm{He}^{*}+\mathrm{He}$ & $5.0 \times 10^{-9} \mathrm{~cm}^{3} / \mathrm{s}$ & 14 \\
\hline H14 & $\mathrm{He}_{2}^{+}+e+\mathrm{He} \rightarrow \mathrm{He}_{2}^{*}+\mathrm{He}$ & $5.0 \times 10^{-27} \mathrm{~cm}^{6} / \mathrm{s}$ & 18 \\
\hline H15 & $\mathrm{He}_{2}^{+}+2 e \rightarrow 2 \mathrm{He}+e$ & $2.0 \times 10^{-20} \mathrm{~cm}^{6} / \mathrm{s}$ & 20 \\
\hline
\end{tabular}

$$
\Gamma_{e}=-\gamma \sum_{p} \Gamma_{+, p},
$$

where $\gamma$ is the secondary emission coefficient. For neutral particles, positive ions, and metastable, the flux at the electrodes is dominated by drift and the diffusive flux is negligible

$$
\frac{\partial n_{+}}{\partial x}=0, \quad \frac{\partial n_{*}}{\partial x}=0 .
$$

Electrons mean energy at the electrode surface is fixed at 0.5 $\mathrm{eV}$.

To facilitate comparison, we have modified our own hydrodynamic model (Ref. 10) so that it becomes comparable to the hybrid model of Eqs. (1) and (2). With the hydrodynamic assumption, reaction rate coefficients, ionization coefficients, drift velocity, and diffusion coefficients can be approximated as a function of the local electric field in the ionized gas between the two electrodes. ${ }^{8-10}$ This eliminates Eq. (1d) from the governing equations of the hydrodynamic model. Again the hydrodynamic model considers six helium species but 15 reactions as detailed in Table II. While different from those of Table I, they represent the current mainstream model for simulation of atmospheric DBD and as such offer a useful base to examine the current understanding against the simulation results using the hybrid model. In the next section, we will comment on the difference in plasma dynamics caused by different reaction choices in Tables I and II.

\section{COMPARISON OF ELECTRICAL PROPERTIES}

We employ the two different plasma models to study a $10 \mathrm{kHz}$ DBD in atmospheric helium with two disk electrodes of $2 \mathrm{~cm}$ radius and an electrode gap of $0.5 \mathrm{~cm}$. The capaci- tance of the dielectric barrier is assumed to be $70 \mathrm{pF}$, and the secondary emission coefficient is fixed at 0.01 . Figure 1 shows the discharge current and various voltages predicted by the hybrid model and the hydrodynamic model. There is a
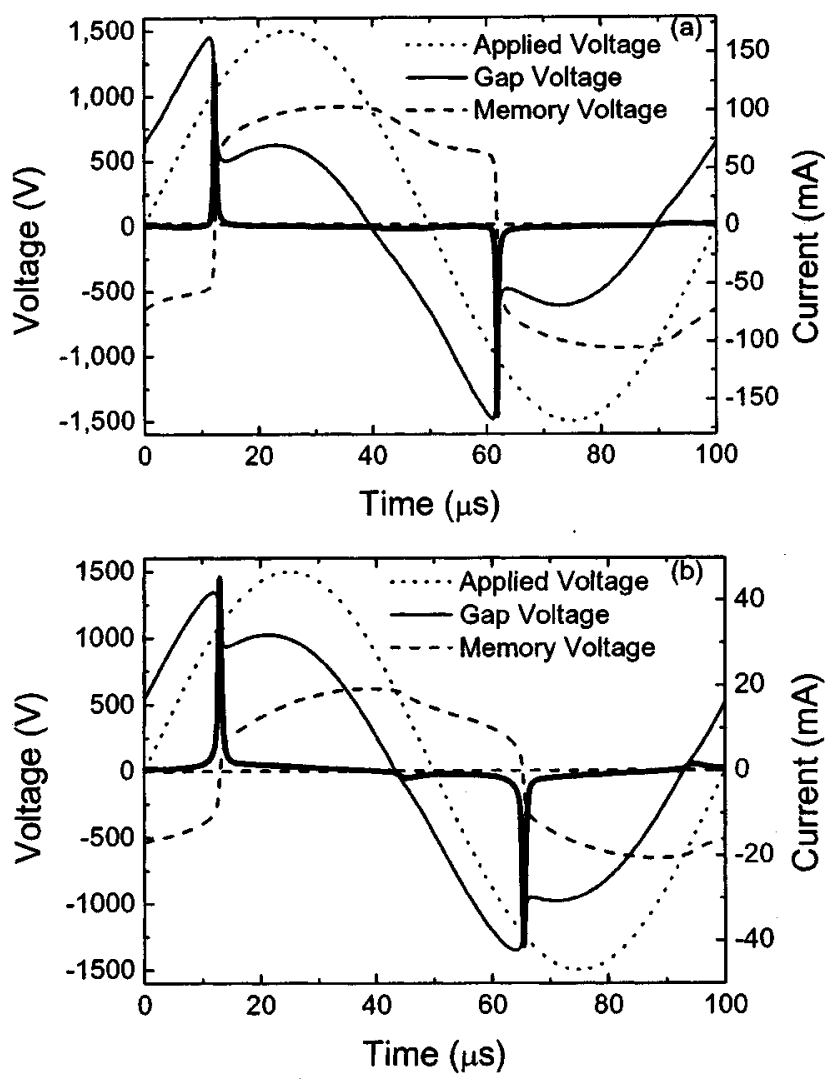

FIG. 1. Discharge current (thick solid curve), applied voltage (dotted curve), gap voltage (thin solid curve), and memory voltage (dashed curve) of a 10 $\mathrm{kHz}$ atmospheric helium DBD predicted by (a) the hybrid model and (b) the hydrodynamic model. 


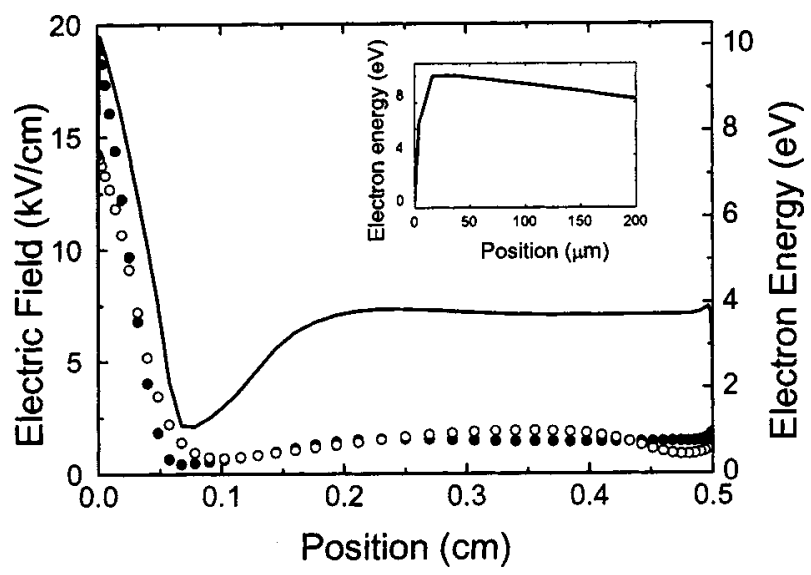

FIG. 2. Comparison of the electric field at the current peak simulated with the hybrid model (solid circles) and that with the hydrodynamic model (hollow circles). Electron mean energy calculated from the hybrid model also at the current peak is added in solid curve.

close agreement in the general current-voltage characteristics predicted by the two different plasma models. In both cases, the typical pattern of one discharge event every half cycle of the applied voltage is apparent and the discharge event occurs approximately $12 \mu \mathrm{s}$ after the applied voltage starts to rise from its zero. The major difference between the hybrid model and the hydrodynamic model is that the discharge current in Fig. 1(a) is $150 \mathrm{~mA}$ whereas in Fig. 1(b) it is 40 $\mathrm{mA}$. The corresponding current density is 12 and $3 \mathrm{~mA} / \mathrm{cm}^{2}$, respectively. As a result, the gap voltage simulated with the hybrid model undergoes a larger drop of some $750 \mathrm{~V}$ during the discharge event.

Figure 2 shows the spatial profiles of the electric field at the current peak computed with the two plasma models. With the hydrodynamic model the peak electric field occurring at the cathode surface is $14.7 \mathrm{kV} / \mathrm{cm}$. This is markedly lower than $19.3 \mathrm{kV} / \mathrm{cm}$ computed with the hybrid model. Using the approximate method of Ref. 12, the hybrid model suggests a sheath thickness of $520 \mu \mathrm{m}$ and the hydrodynamic model yields a larger sheath thickness of $640 \mu \mathrm{m}$. According to the relationship between sheath electric field and sheath thickness, ${ }^{12}$ a larger sheath thickness should correspond to a smaller electric field and comparison of the two electric field profiles in Fig. 2 confirms this correlation.

A key advantage of the hybrid model is that it provides information on electron kinetics, and as shown in Fig. 2, the electron mean energy reaches its maximum value of 10.15 $\mathrm{eV}$ at $36 \mu \mathrm{m}$ from the cathode surface. Over this short distance from the cathode surface, electrons are not in equilibrium with the local electric field but are accelerated to toward their eventual equilibrium. Moving away from its peak toward the plasma bulk, the electron mean energy falls off in a similar way to the electric field and reaches a minimum of $1.1 \mathrm{eV}$ around $670 \mu \mathrm{m}$ from the cathode. This electron energy drop is a result of frequent electron ionization of helium atoms in the sheath region until most electrons are no longer sufficiently energetic for further ionization. At this instant, the boundary of the sheath with the plasma bulk is reached again at $670 \mu \mathrm{m}$ from the cathode. Subsequently as they travel into the positive column, most electrons undergo a net
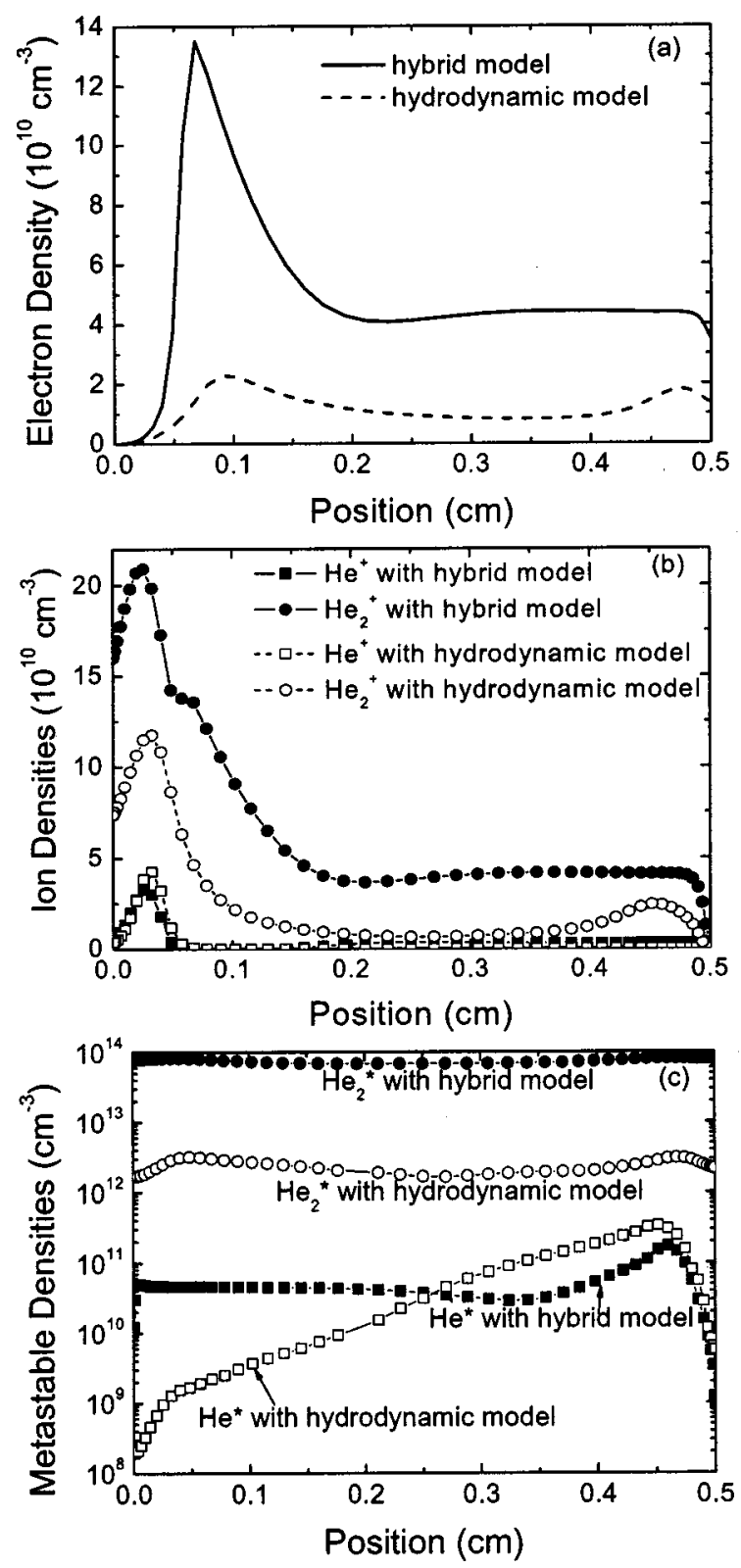

FIG. 3. Comparison of the hybrid simulation results (solid curve or solid markers) and hydrodynamic simulation results (dashed curve or hollow markers) at the peak of the discharge current for (a) electron density, (b) ion densities, and (c) metastable densities.

acceleration by the local electric field and gain kinetic energy leading to increased electron mean energy. Upon their arrival at the positive column, electrons reach an approximately constant mean energy of about $3.8 \mathrm{eV}$. This net acceleration is significant, partly contributing to the larger discharge current in Fig. 1 as compared to that calculated with the hydrodynamic model.

Figure 3 shows particle densities calculated with the two plasma models when the discharge current is the largest and the cathode is on the left hand side. In general the hybrid model yields greater particle densities. Spatial profile of electron density is seen to be similar regardless of the plasma model used. However the hybrid model suggests a maximum electron density of $1.35 \times 10^{11} \mathrm{~cm}^{-3}$ at $650 \mu \mathrm{m}$ from the cathode whereas with the hydrodynamic model the maximum electron density is found to be $3.32 \times 10^{10} \mathrm{~cm}^{-3}$ at 780 
$\mu \mathrm{m}$ away from the cathode. So the maximum electron density calculated with the hybrid model is 4.1 times greater. This compares closely to 3.8 , the ratio of the peak discharge currents in Fig. 1 calculated with the two different plasma models. For molecular helium ions, the hybrid model results in a peak density of $2.1 \times 10^{11} \mathrm{~cm}^{-3}$ and the hydrodynamic model $1.3 \times 10^{11} \mathrm{~cm}^{-3}$, thus a ratio of 1.6 times as shown in Fig. 3(b). Interestingly for atomic helium ions, the hybrid model finds their peak density at $3.3 \times 10^{10} \mathrm{~cm}^{-3}$ lower than $4.9 \times 10^{10} \mathrm{~cm}^{-3}$ obtained from the hydrodynamic model. Their spatial profiles obtained with the two methods are nevertheless very similar. For helium molecular metastables, both models suggest a relative flat density distribution in Fig. 3(c). Again the hybrid model suggests a larger density of $8.2 \times 10^{13} \mathrm{~cm}^{-3}$, a factor of 28 above $2.9 \times 10^{12} \mathrm{~cm}^{-3}$ calculated with the hydrodynamic model. For atomic helium metastables this ratio is smaller at 4.9.

From the above comparison between the two plasma models, it is evident that under the same operation conditions the hybrid model suggests more significant gas ionization than the hydrodynamic model, thus yielding larger densities of electrons, all helium ions and the dominating molecular helium metastables. Given that the hydrodynamic model does not account for the electron nonequilibrium with the local electric field in the sheath region, its comparison with the hybrid model suggests that it underestimates gas ionization thus resulting in an underestimate of the production of both charged particles and metastables. From the standpoint of applications, the hydrodynamic model is likely to underestimate the extent of plasma chemistry for a given set of operation parameters. Also its underestimate of electron density suggests that it may overestimate the parametric range for stable plasma before the glow-to-arc transition is reached.

It is possible that the difference in results of the two plasma models may be due to different reactions and reaction rates (see Tables I and II). To this end, we have developed a second hydrodynamic model with the exact set of reactions used in the hybrid model. To allow for a hydrodynamic treatment, we use space-averaged electron mean energy from Fig. 2 to obtain a constant rate for all nine reactions in Table I and retain the field-dependent ionization and excitation coefficients of Ref. 8. This is intended to contrast out the difference between two reaction sets of Tables I and II used in the two plasma models. Numerical calculation using this second hydrodynamic model results in a peak discharge current of 8 $\mathrm{mA}$, smaller than $40 \mathrm{~mA}$ of Fig. 1(b). Therefore hydrodynamic models indeed underestimate gas ionization. Comparison in Figs. 1-3 employs the first hydrodynamic model of Table II, because this model reflects the nature of most current hydrodynamic models.

\section{SHEATH CHARACTERISTICS}

It has been established in Fig. 2 that electrons are not in equilibrium with the local electric field in the sheath region. In the case of Fig. 2, the electron mean energy reaches its peak of $9 \mathrm{eV}$ at $36 \mu \mathrm{m}$ from the cathode when the peak discharge current density is $150 / \pi \times 2^{2}=12 \mathrm{~mA} / \mathrm{cm}^{2}$. At this point, the peak electric field is $19.3 \mathrm{kV} / \mathrm{cm}$ at the cathode

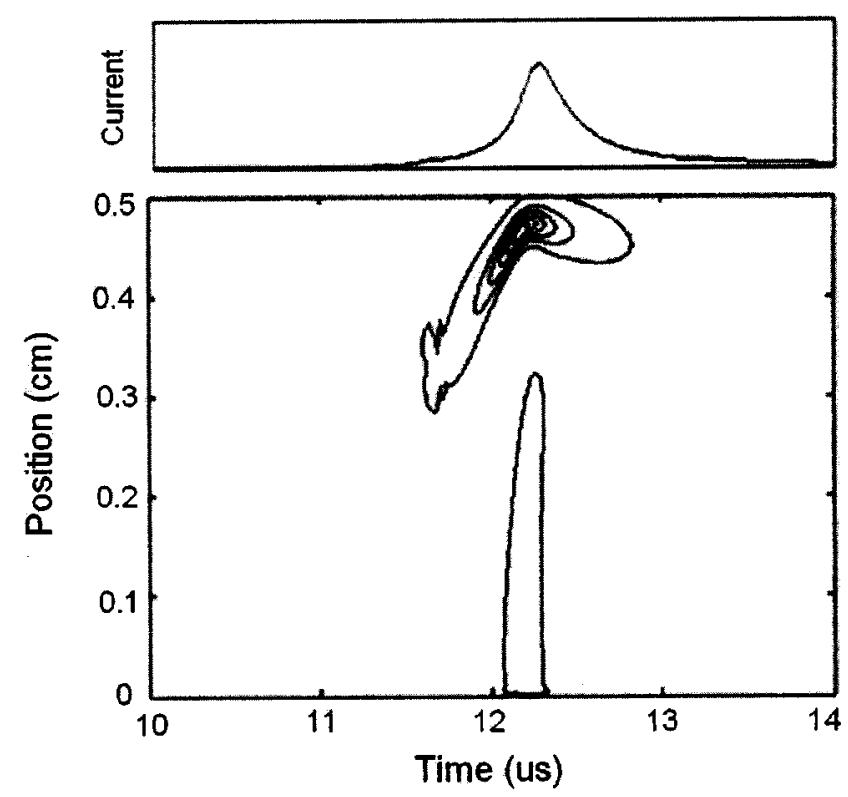

FIG. 4. Spatiotemporal profile of net electron production rate (lower graph) over the same period of the discharge current (upper graph). The maximum electron production rate is $3.9 \times 10^{18} \mathrm{~cm}^{-3} \mathrm{~s}^{-1}$, and the cathode is at $0.5 \mathrm{~cm}$.

surface and the sheath thickness is $520 \mu \mathrm{m}$. For dc APGD in helium, a current density of $12 \mathrm{~mA} / \mathrm{cm}^{2}$ corresponds to a sheath thickness of $150 \mu \mathrm{m}$, a peak sheath field of $11 \mathrm{kV} / \mathrm{cm}^{2}$, and a maximum electron mean energy of 24 eV. ${ }^{12}$ Therefore compared to dc APGD at the same current density, atmospheric DBD have much less energetic electrons in the sheath region even though its peak electric field is larger. This is because sheath electrons are permanently accelerated in dc APGD and they reach the helium ionization energy over a much shorter distance. By contrast the oscillating applied voltage in atmospheric DBD establishes a sheath region near one electrode during one half-cycle and then destroys it before setting up another sheath region near the other electrode during the next half-cycle. As a result, sufficient electron acceleration for gas ionization must be achieved over a greater sheath region in atmospheric DBD. It is worth mentioning that the dynamic sheath establishment and destruction in atmospheric DBD is useful for control of the glow-to-arc transition. In the case of radio-frequency APGD at $13.56 \mathrm{MHz}$, a discharge current density of $12 \mathrm{~mA} / \mathrm{cm}^{2}$ would lead to a peak electron mean energy of 5 $\mathrm{eV}$ and a sheath region comparable to the gap size. ${ }^{21}$ Therefore as the excitation frequency increases and at the same discharge current density, the peak electron mean energy decreases and the sheath thickness increases.

Sheath dynamics may be seen from spatiotemporal profile of gas ionization rate as shown in Fig. 4. It is evident that sheath establishment coincides the discharge event of some 1 $\mu$ s duration. The most significant ionization occurs near the cathode although ionization in the positive column is not insignificant. The latter is related to the large electron mean energy of $3.9 \mathrm{eV}$ in Fig. 2 even though the electric field is very small in the positive column. The short duration of the rapid electron production from 11.5 to $12.5 \mu$ s reflects the short time scale, with respect to the excitation period, over which the gap voltage is held above the breakdown voltage. 

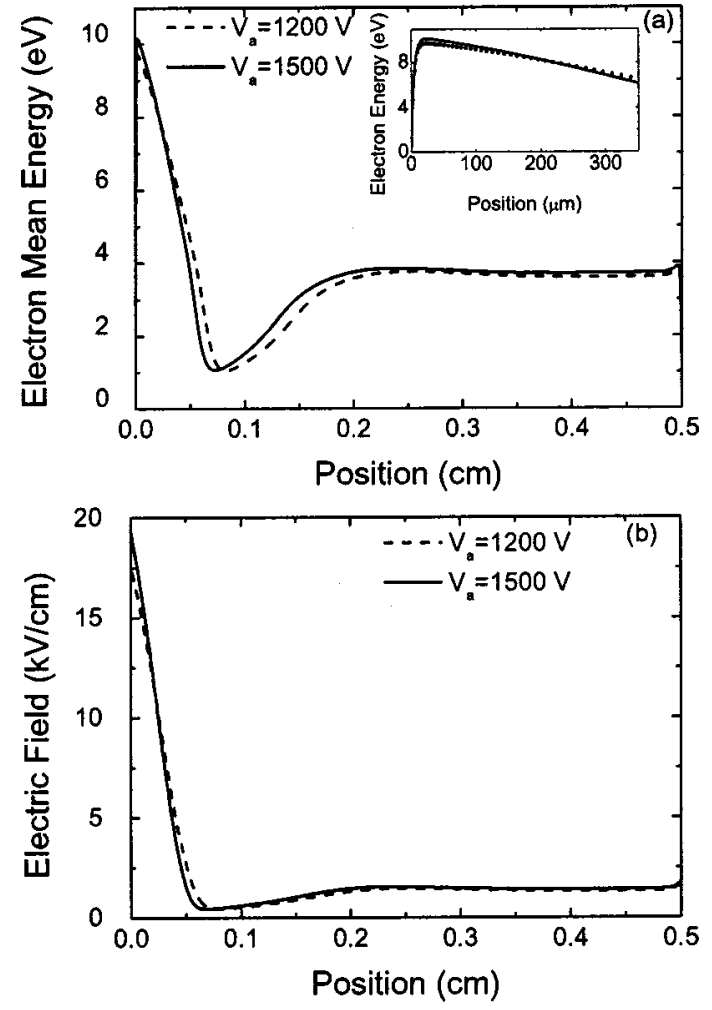

FIG. 5. Spatial dependence at the current peak of (a) electron mean energy and (b) at two different levels of the applied voltage.

This is in sharp contrast with rf APGD for which significant gas ionization occurs over a time scale comparable to the excitation period. After $12.5 \mu \mathrm{s}$, no significant ionization can be sustained and the sheath region relaxes until the subsequent discharge current is triggered in the next half-cycle. Therefore the sheath undergoes establishment, relaxation, and reestablishment periodically.

Operation range of atmospheric DBD is small compared to dc APGD and rf APGD. Figure 5 shows spatial profiles of electron mean energy and electric field at two different amplitudes of the applied voltage. If the amplitude of the applied voltage is reduced below $1100 \mathrm{~V}$, it becomes difficult to sustain any stable atmospheric DBD whereas an applied voltage with amplitude above $1800 \mathrm{~V}$ leads to multiple discharge peaks every half-cycle. Yet Fig. 5 suggests that the electron mean energy and the electric field do not change significantly over this range of the applied voltage, perhaps a result of a self-adjustment between the applied voltage and the memory voltage. Again this is different from rf APGD for which electron mean energy and electric field can change over a much greater range. ${ }^{21}$ Figure 5 also suggests that a smaller electron mean energy correlates to a broader sheath thickness, similar to that in dc APGD (Ref. 12) and rf APGD. $^{21}$

\section{CONCLUSION}

We employed an electron-hybrid model to model atmospheric DBD and found that results predicted by the usual hydrodynamic model underestimate the level of gas ionization and the discharge current. It has been shown that electrons are not in equilibrium with the local electric field for a significant part of the sheath region, and as such it is important to account for this nonequilibrium. Comparison with dc APGD and radio-frequency APGD suggests that as the excitation frequency increases the electron mean energy decreases and the sheath thickness increases. Gas ionization was found to be significant over a short time scale of $1 \mu \mathrm{s}$, a very small fraction of the excitation period. It was also found that variation in the amplitude of the applied voltage does not significantly alter sheath characteristics in terms of the peak electric field and the peak electron mean energy, reflecting a self-adjustment between the applied voltage and the memory voltage.

${ }^{1}$ B. Eliasson and U. Kogelschatz, IEEE Trans. Plasma Sci. 19, 1063 (1991).

${ }^{2}$ A. Schutze, J. Y. Jeong, S. E. Babayan, J. Park, and G. S. Selwyn, IEEE Trans. Plasma Sci. 26, 1685 (1998).

${ }^{3}$ T. Yokoyama, M. Kogoma, T. Moriwaki, and S. Okazaki, J. Phys. D 23, 1125 (1990).

${ }^{4}$ R. H. Stark and K. H. Schoenbach, J. Appl. Phys. 85, 2075 (1999).

${ }^{5}$ T. C. Montie, K. Kelly-Wintenberg, and J. R. Roth, IEEE Trans. Plasma Sci. 28, 41 (2000).

${ }^{6}$ M. J. Shenton and G. C. Stevens, J. Phys. D 34, 2761 (2001).

${ }^{7}$ E. E. Kunhardt, IEEE Trans. Plasma Sci. 28, 189 (2000).

${ }^{8}$ F. Massines, A. Rabehi, Ph. Decomps, R. B. Gadri, P. Segur, and C. Mayoux, J. Appl. Phys. 83, 2950 (1998).

${ }^{9}$ Yu. B. Golubovskii, V. A. Maiorov, J. Behnke, and J. F. Behnke, J. Phys. D 35, 751 (2002).

${ }^{10}$ M. G. Kong and X. T. Deng, IEEE Trans. Plasma Sci. 31, 7 (2003).

${ }^{11}$ X. Yuan and L. L. Raja, IEEE Trans. Plasma Sci. 31, 495 (2003).

${ }^{12}$ J. Shi and M. G. Kong, J. Appl. Phys. 94, 5504 (2003).

${ }^{13}$ A. V. Phelps, Plasma Sources Sci. Technol. 10, 329 (2001).

${ }^{14}$ J. M. Pouvesle, A. Bouchoule, and J. Stevefelt, J. Chem. Phys. 77, 817 (1982).

${ }^{15}$ R. Deloche, P. Monchicourt, M. Cheret, and F. Lambert, Phys. Rev. A 13, 1140 (1976).

${ }^{16}$ G. Myers and J. Cunningham J. Chem. Phys. 67, 3352 (1977).

${ }^{17}$ T. Quinteros, H. Gao, D. R. DeWitt, R. Schuch, S. Pajek, S. Asp, and D. Belkic, Phys. Rev. A 51, 1340 (1995).

${ }^{18}$ P. C. Hill and P. R. Herman, Phys. Rev. A 47, 4837 (1993).

${ }^{19}$ C. B. Collins, H. S. Hicks, W. E. Wells, and R. Burton, Phys. Rev. A 6, 1545 (1972).

${ }^{20}$ J. Berlande, M. Cheret, R. Deloche, A. Gonfalone, and C. Mans, Phys. Rev. A 1, 887 (1970).

${ }^{21}$ J. J. Shi and M. G. Kong, J. Appl. Phys. 97, 023306 (2005). 